\title{
Localized subacute thyroiditis presenting as a painful hot nodule
}

\author{
Lian-Xi Li ${ }^{i *}$, Xing $\mathrm{Wu}^{2+}$, Bing Hu${ }^{2}$, Hui-Zhen Zhang ${ }^{3}$ and Han-Kui Lu ${ }^{4 *}$
}

\begin{abstract}
Background: A diagnosis of subacute thyroiditis is readily considered when patients present with a particular set of typical clinical characteristics. Subacute thyroiditis sometimes presents as a solitary cold nodule; however, the presence of a hot nodule in patients with subacute thyroiditis is exceedingly rare.

Case presentation: Here, the case of a 57-year-old woman complaining of pain in the left neck and fatigue for two weeks is presented. Physical examination revealed a painful and tender nodule with a diameter of approximately $1.5 \mathrm{~cm}$ in the left neck, although all laboratory tests, including white blood cell count, neutrophil percentage, erythrocyte sedimentation rate (ESR), thyroid function, and thyroglobin levels, were normal. A neck ultrasound revealed a hypoechoic mass $(1.5 \times 0.8 \mathrm{~cm})$ in the left thyroid, and thyroid scintigraphy of the left thyroid with Technetium-99 m (99 m-Tc) demonstrated a focal accumulation of radiotracer. Furthermore, fine-needle aspiration biopsy from the nodule revealed the presence of multinuclear giant cells. The patient was well; there was no cervical mass detected upon palpation following two months of prednisone treatment, and follow-up ultrasound screening and scintigraphy demonstrated the disappearance of the nodule.
\end{abstract}

Conclusion: This case, presenting with a localized painful hot nodule, normal thyroid function, normal ESR, and normal serum thyroglobulin levels, is a rare case of subacute thyroiditis, which should be considered during differential diagnosis.

Keywords: Subacute thyroiditis, Thyroid nodule, Hot nodule

\section{Background}

Subacute thyroiditis, also known as de Quervain's thyroiditis, giant-cell thyroiditis, or subacute granulomatous thyroiditis, is a spontaneously remitting inflammatory disease of the thyroid gland $[1,2]$. Subacute thyroiditis is generally caused by viral infection and is the most common cause of a painful thyroid [1,3]. Patients with subacute thyroiditis usually have a history of antecedent viral infection and subsequently suffer from neck pain, thyroid tenderness, fever, and fatigue. Upon physical examination, the thyroid of the patient is often tender and diffusely enlarged.

\footnotetext{
* Correspondence: lilx@sjtu.edu.cn; luhankui@sina.com

†Equal contributors

'Department of Endocrinology and Metabolism, Shanghai Diabetes Institute; Shanghai Clinical Center for Diabetes; Shanghai key Laboratory of Diabetes Mellitus, Shanghai Jiao Tong University Affiliated Sixth People's Hospital, 600 Yishan Road, Shanghai 200233, China

${ }^{4}$ Department of Nuclear Medicine, Shanghai Jiao Tong University Affiliated Sixth People's Hospital, 600 Yishan Road, Shanghai 200233, China

Full list of author information is available at the end of the article
}

In most cases, a diagnosis of subacute thyroiditis is usually self-evident and can be made based on patient history, physical and laboratory findings, and the clinical course of the disease. In some cases, in addition to the clinical course and features, fine needle aspiration cytology, ultrasound, and scintigraphy analyses may support the diagnosis of subacute thyroiditis. For example, thyroid radioisotope scanning generally demonstrates a low uptake of Technetium-99 m (99 m-Tc) or 131I [4]. However, patients with subacute thyroiditis sometimes present with puzzling clinical features that can escape early recognition $[2,3,5,6]$. Here, a patient with subacute thyroiditis, who presented with a solitary painful thyroid nodule in the absence of typical laboratory test characteristics that would suggest subacute thyroiditis and whose $99 \mathrm{~m}$-Tc thyroid scan revealed a hot nodule in the left lobe of thyroid, is described. To the best of our knowledge, the presence of a hot nodule in a patient with subacute thyroiditis has not been previously reported.

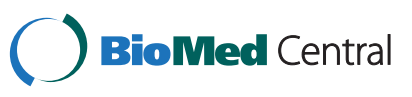

(c) 2014 Li et al.; licensee BioMed Central Ltd. This is an open access article distributed under the terms of the Creative Commons Attribution License (http://creativecommons.org/licenses/by/2.0), which permits unrestricted use, distribution, and reproduction in any medium, provided the original work is properly cited. 


\section{Case presentation}

A 57-year-old woman with no history of thyroid disease visited our outpatient endocrine clinic on July 27, 2012. Two weeks prior, she had developed symptoms of pain in the left neck and fatigue. Physical examination revealed a focal nodule of the left thyroid lobe that had a diameter of approximately $1.5 \mathrm{~cm}$ without local redness or lymph node enlargement, which was painful and tender upon examination. There was no fever or signs of hyperthyroidism such as tachycardia, insomnia, or tremors. The patient's thyroid function tests were normal (thyroid stimulating hormone $=1.25 \mathrm{mU} / \mathrm{L}$, normal range: $0.27-4.2 \mathrm{mU} / \mathrm{L}$; free triiodothyronine $=5.59 \mathrm{pmmol} / \mathrm{L}$, normal range: $3.1-6.8 \mathrm{pmmol} / \mathrm{L}$; free thyroxine $=18.80$ $\mathrm{pmmol} / \mathrm{L}$, normal range: $12-22 \mathrm{pmmol} / \mathrm{L}$ ) and tests for anti-thyroglobulin $(<10 \mathrm{KIU} / \mathrm{L}$, normal range: $0-115$ $\mathrm{KIU} / \mathrm{L})$, anti-thyroid peroxidase (10.54 KIU/L, normal range: $0-35 \mathrm{KIU} / \mathrm{L})$, and anti-thyrotropin-receptor antibodies $(<1 \mathrm{U} / \mathrm{L}$, normal range: $0-2 \mathrm{U} / \mathrm{L})$ were negative. The patient's serum thyroglobulin levels were normal (61.32 ug/L, normal range: $1.40-78 \mathrm{ug} / \mathrm{L})$; her white cell count was $5.2 \times 10^{9} / \mathrm{L}$ with a normal differential, and her erythrocyte sedimentation rate (ESR) was $20 \mathrm{~mm} /$ hour (normal range: $0-38 \mathrm{~mm} / \mathrm{h}$ ).

A thyroid ultrasound examination revealed a dyshomogeneous and hypoechoic mass $(1.5 \times 0.8 \mathrm{~cm})$ in the left thyroid lobe that exhibited an irregular and poorly defined border (Figure 1A). Thyroid scintigraphy with $99 \mathrm{~m}$-Tc demonstrated a focal accumulation of radiotracer uptake in the lower part of the left thyroid lobe but a normal uptake and configuration of the middle and upper portion of the left lobe and the right lobe (Figure 1B). Fine-needle aspiration biopsy from the nodule in the lower left lobe revealed multinuclear giant cells consistent with subacute thyroiditis (Figure 2). Subsequently, localized subacute thyroiditis was suspected and the patient was put on prednisone ( $30 \mathrm{mg} /$ day) for 10 days, which resulted

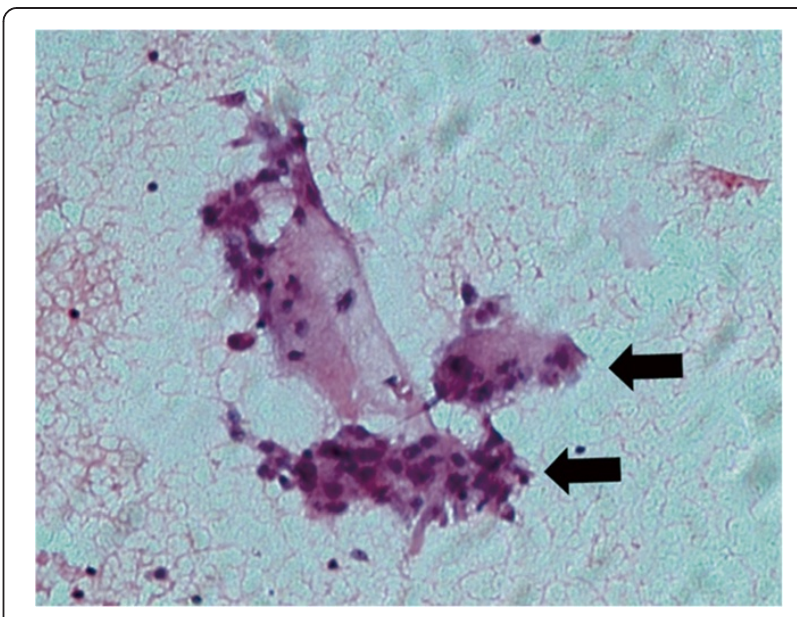

Figure 2 Fine-needle aspiration cytology of the left thyroid nodule. Fine-needle aspiration biopsy from the nodule in the left lobe revealed multinuclear giant cells in the thyroid nodule consistent with subacute thyroiditis (indicated by black arrows).

in a rapid resolution of the neck pain. Within a week, the patient's fatigue had disappeared and the tender thyroid nodule had regressed. Prednisone was gradually withdrawn and ultimately stopped after 2 months at which time palpation did not demonstrate a cervical mass. Repeated ultrasound screening revealed a disappearance of the hypoechoic nodule (Figure 3A) and follow-up scintigraphy analysis found the thyroid exhibited an even distribution of radionuclide in both lobes (Figure 3B).

\section{Conclusions}

Subacute thyroiditis is the most common cause of nonautoimmune thyroiditis [7]. In addition to the typical clinical signs, characteristic ultrasound findings of subacute thyroiditis include the presence of an ill-defined hypoechoic area with a nonhomogeneous pattern [8]. Recently, Ruchala et al. [9] demonstrated the usefulness of

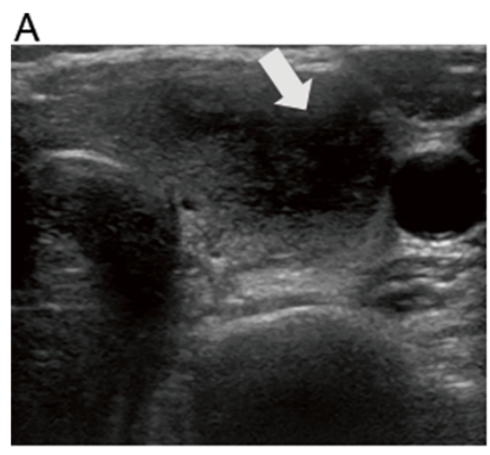

\section{B}

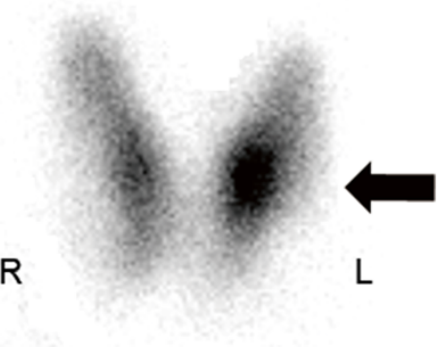

\section{Before treatment}

Figure 1 Initial thyroid ultrasound scanning and $99 \mathrm{~m}$-Technetium scintiscan. A. Thyroid ultrasonography revealed a dyshomogeneous and hypoechoic nodule $(15 \times 0.8 \mathrm{~mm})$ with an irregular and poorly defined border in the left thyroid lobe (indicated by a white arrow). B. Thyroid scintigraphy with $99 \mathrm{~m}$-Tc showed a focal accumulation of radiotracer uptake in the lower lobe of the left thyroid, which represents the palpable tender nodule (indicated by a black arrow). 


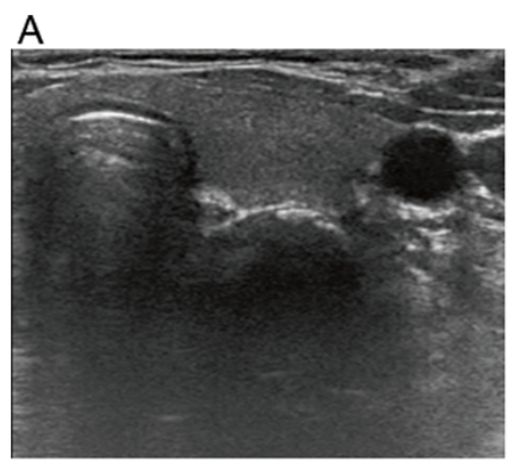

B

\section{After treatment}

Figure 3 Follow-up ultrasound scanning and $\mathbf{9 9} \mathbf{m}$-Technetium scintiscan. A. Follow-up ultrasound scanning demonstrated the disappearance of the hypoechoic nodule in the left thyroid lobe. B. Follow-up scintigraphy showed the disappearance of the "hot" nodule in the left thyroid lobe and an even distribution of radionuclide in both lobes.

sonoelastography for the diagnosis of subacute thyroiditis. The cytological features found during thyroid fine-needle aspiration include the presence of large multinucleated giant cells or epithelioid granulomas, but the absence of these findings does not exclude the diagnosis of subacute thyroiditis $[10,11]$. Generally speaking, elevated serum thyroid hormones, a tender enlarged thyroid, and low radioiodine thyroid uptake are characteristic of subacute thyroiditis [12]. Although cases with these typical signs may present little difficulty for a diagnosis of subacute thyroiditis, this disorder does not always present in a classic fashion and may lead to difficulties during diagnosis $[5,6]$. Sometimes the diagnosis may be less clear, particularly when the primary presenting symptom is a solitary thyroid nodule in conjunction with normal thyroid function, thyroglobulin levels, and a normal ESR [13].

Previously, thyroid nodules have been identified in association with subacute thyroiditis and, in some patients with subacute thyroiditis, only one nodule is present $[14,15]$. For example, Liel [16] reported a case that presented with the coexistence of subacute thyroiditis and an autonomously functioning thyroid nodule. More often, localized forms of subacute thyroiditis present as painful and tender "cold" thyroid nodules, which disappear following recovery [14]. However, subacute thyroiditis that presents as a painful "hot" nodule is exceedingly rare and has not been reported. In this case, laboratory tests, including white blood cell count, neutrophil percentage, thyroid function, thyroglobin levels, and ESR, were normal and non-diagnostic but the clinical findings (neck pain, thyroid tenderness, and fatigue) led to the consideration of a diagnosis of subacute thyroiditis. Therefore, further work-ups were completed including an ultrasound examination of the neck, thyroid scintigraphy with $99 \mathrm{~m}$-Tc, and fine needle aspiration cytology of the nodule. Ultrasound examination demonstrated a dyshomogeneous and hypoechoic mass in the thyroid, which was characteristic of subacute thyroiditis, and thyroid scintigraphy showed a focal accumulation of radiotracer uptake in the thyroid nodule. The histological features of the nodule were also typical of subacute thyroiditis. Therefore, a diagnosis of localized subacute thyroiditis was given and the patient was prescribed prednisone, which resulted in the disappearance of the hot thyroid nodule. The treatment of subacute thyroiditis is essentially symptomatic and includes nonsteroidal anti-inflammatory agents or, occasionally, glucocorticoids if the symptoms are prolonged or severe. In the current case, treatment with steroids resulted in an amelioration of the patient's symptoms and the disappearance of the thyroid nodule after 2 months.

Based on the course and the clinical presentation of the present case, a diagnosis of subacute thyroiditis could be established. The disappearance of the thyroid nodule following prednisone treatment further confirms the diagnosis of subacute thyroiditis following presentation with a thyroid hot nodule. Here, the appearance of the hot thyroid nodule was unusual in that it did not show the usual pattern of low uptake during radioisotope scanning. This case demonstrates that subacute thyroiditis may present as a solitary painful hot nodule in conjunction with normal thyroid function, thyroglobulin levels, and ESR and should, therefore, be considered in the differential diagnosis of such lesions.

The mechanism of $99 \mathrm{~mm}$-Tc localization in subacute thyroiditis is not known. Tonami et al. [17] reported two cases of subacute thyroiditis in which thyroid scintigrams with 201TI chloride showed increased radionuclide activity in the affected areas but decreased activity in the affected areas following thyroid scintigrams with $99 \mathrm{~m}$-Tc. It was presumed that this is primarily due to increased membrane permeability in the inflammatory lesion without the apparent destruction of the thyroid gland, which is typically indicated by normal thyroglobulin levels and thyroid function in the patient. Therefore, 
the present case suggests the clinical and pathological heterogeneity of subacute thyroiditis.

In conclusion, this case demonstrates that subacute thyroiditis should be considered as a differential diagnosis following presentation with a solitary painful thyroid hot nodule in conjunction with normal thyroid function, thyroglobulin levels, and ESR. Additionally, this case emphasizes the heterogeneous pattern of thyroid imaging in subacute thyroiditis.

\section{Consent}

Written informed consent was obtained by the patient for the publication of this case report and any accompanying images.

\section{Competing interests}

The authors declare that they have no competing interests.

\section{Authors' contributions}

LL drafted the manuscript; WX collected all medical reports of the patients and LHK revised the manuscript critically. HB performed ultrasound examination and ZHZ carried out pathological examination. All authors read and approved the final manuscript.

\section{Acknowledgments}

This work was supported by grants from the National Natural Science Foundation of China (81170759) and Innovation Program of Shanghai Municipal Education Commission (1322015).

\section{Author details}

${ }^{1}$ Department of Endocrinology and Metabolism, Shanghai Diabetes Institute; Shanghai Clinical Center for Diabetes; Shanghai key Laboratory of Diabetes Mellitus, Shanghai Jiao Tong University Affiliated Sixth People's Hospital, 600 Yishan Road, Shanghai 200233, China. 'Department of Ultrasonography, Shanghai Jiao Tong University Affiliated Sixth People's Hospital, 600 Yishan Road, Shanghai 200233, China. ${ }^{3}$ Department of Pathology, Shanghai Jiao Tong University Affiliated Sixth People's Hospital, 600 Yishan Road, Shanghai 200233, China. ${ }^{4}$ Department of Nuclear Medicine, Shanghai Jiao Tong University Affiliated Sixth People's Hospital, 600 Yishan Road, Shanghai 200233, China.

Received: 29 July 2013 Accepted: 31 December 2013

Published: 8 January 2014

\section{References}

1. Pearce EN, Farwell AP, Braverman LE: Thyroiditis. N Engl J Med 2003, 348:2646-2655.

2. Geva T, Theodor R: Atypical presentation of subacute thyroiditis. Arch Dis Child 1988, 63:845-846.

3. Tsai CH, Lee JJ, Liu CL, Tzen CY, Cheng SP: Atypical subacute thyroiditis. Surgery 2010, 147:461-462.

4. Kitchener MI, Chapman IM: Subacute thyroiditis: a review of 105 cases. Clin Nucl Med 1989, 14:439-442.

5. Huang C, Wang X: Subacute thyroiditis manifesting as a thyroid mass, vocal cord paralysis, and hypercalcemia. Endocr Pract 2012, 18:e17-e20.

6. Bartels PC, Boer RO: Subacute thyroiditis (de Quervain) presenting as a painless "cold" nodule. J Nucl Med 1987, 28:1488-1490.

7. Bianda T, Schmid C: De Quervain's subacute thyroiditis presenting as a painless solitary thyroid nodule. Postgrad Med J 1998, 74:602-603.

8. Park SY, Kim EK, Kim MJ, et al: Ultrasonographic characteristics of subacute granulomatous thyroiditis. Korean J Radiol 2006, 7:229-234.

9. Ruchala M, Szczepanek E, Sowinski J: Sonoelastography in de Quervain thyroiditis. J Clin Endocrinol Metab 2011, 96:289-290.

10. Mordes DA, Brachtel EF: Cytopathology of subacute thyroiditis. Diagn Cytopathol 2012, 40:433-434.
11. Garcia SJ, Gimenez BA, Sola PJ, et al: Fine-needle aspiration of subacute granulomatous thyroiditis (De Quervain's thyroiditis): a clinico-cytologic review of 36 cases. Diagn Cytopathol 1997, 16:214-220.

12. Sari O, Erbas B, Erbas T: Subacute thyroiditis in a single lobe. Clin Nucl Med 2001, 26:400-401.

13. Szczepanek-Parulska E, Zybek A, Biczysko M, Majewski P, Ruchala M: What might cause pain in the thyroid gland? Report of a patient with subacute thyroiditis of atypical presentation. Endokrynol Pol 2012, 63:138-142.

14. Hardoff R, Baron E, Sheinfeld M, Luboshitsky R: Localized manifestations of subacute thyroiditis presenting as solitary transient cold thyroid nodules. A report of 11 patients. Clin NuCl Med 1995, 20:981-984.

15. Nygaard B, Jarlov AE, Hegedus L, Schaadt B, Kristensen LO, Hansen JM: Long-term follow-up of thyroid scintigraphies after 131I therapy of solitary autonomous thyroid nodules. Thyroid 1994, 4:167-171.

16. Liel $Y$ : The survivor: association of an autonomously functioning thyroid nodule and subacute thyroiditis. Thyroid 2007, 17:183-184.

17. Tonami N, Bunko H, Kuwajima A, Hisada K: Increased localization of 201 Tl-chloride in subacute thyroiditis. Clin Nucl Med 1979, 4:3-5.

doi:10.1186/1472-6823-14-4

Cite this article as: Li et al.: Localized subacute thyroiditis presenting as

a painful hot nodule. BMC Endocrine Disorders 2014 14:4.

\section{Submit your next manuscript to BioMed Central and take full advantage of:}

- Convenient online submission

- Thorough peer review

- No space constraints or color figure charges

- Immediate publication on acceptance

- Inclusion in PubMed, CAS, Scopus and Google Scholar

- Research which is freely available for redistribution 